STRUCTURE REPORTS

ISSN 1600-5368

Received 31 August 2014

Accepted 14 September 2014

Edited by $\mathrm{H}$. Stoeckli-Evans, University of Neuchâtel, Switzerland

Keywords: crystal structure; 2-alkoxy-

benzamide; hydrogen bonding.

CCDC reference: 1024316

Supporting information: this article has supporting information at journals.iucr.org/e

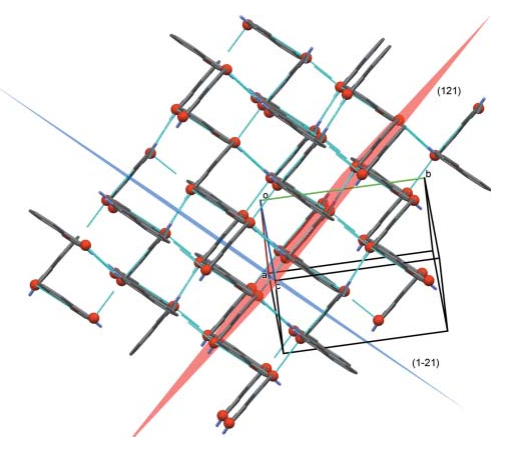

OPEN $\odot$ ACCESS

\section{Crystal structure of 2-pentyloxybenzamide}

\author{
Bernhard Bugenhagen, ${ }^{\mathrm{a}}$ Yosef $\mathrm{Al}$ Jasem $^{\mathrm{b}}$ and Thies Thiemann ${ }^{\mathrm{c} *}$
}

${ }^{\mathbf{a}}$ Institute of Inorganic Chemistry, University of Hamburg, Hamburg, Germany, ${ }^{\mathbf{b}}$ Department of Chemical Engineering,
United Arab Emirates University, AL Ain, Abu Dhabi, United Arab Emirates, and ${ }^{\mathbf{c}}$ Department of Chemistry, United Arab
Emirates University, AL Ain, Abu Dhabi, United Arab Emirates. *Correspondence e-mail: thies@uaeu.ac.ae

In the title molecule, $\mathrm{C}_{12} \mathrm{H}_{17} \mathrm{NO}_{2}$, the amide $\mathrm{NH}_{2}$ group is oriented toward the pentyloxy substituent and an intramolecular $\mathrm{N}-\mathrm{H} \cdots \mathrm{O}$ hydrogen bond is formed with the pentyloxy $\mathrm{O}$ atom. The benzene ring forms dihedral angles of $2.93(2)$ and $5.60(2)^{\circ}$ with the amide group and the pentyloxy group mean planes, respectively. In the crystal, molecules are linked by pairs of $\mathrm{N}-\mathrm{H} \cdots \mathrm{O}$ hydrogen bonds, forming inversion dimers with their molecular planes parallel, but at an offset of 0.45 (1) $\AA$ to each other. These dimers are ordered into two types of symmetry-related columns extended along the $a$ axis, with the mean plane of one set of dimers in a column approximately parallel to (121) and the other in a column approximately parallel to (121). The two planes form a dihedral angle of $85.31(2)^{\circ}$, and are linked via $\mathrm{C}-\mathrm{H} \cdots \mathrm{O}$ hydrogen bonds and $\mathrm{C}-\mathrm{H} \cdots \pi$ interactions, forming a three-dimensional framework structure.

\section{Chemical context}

2-Alkoxybenzamide moieties can be found as structural units in medicinally active compounds, such in dopamine (DA) receptor antagonists (van de Waterbeemd \& Testa, 1983). Typically such components are Sulpiride ${ }^{\mathbb{R}}$, Metoclopramide $^{\circledR}$ and Tiapride ${ }^{\mathbb{R}}$. Other substituted 2-alkoxybenzamides have been found to be antagonists of chemotherapy-induced nausea (Monkovic et al., 1988). Also, 2-alkoxybenzamides have been proposed as agonists of the $\alpha 7$ nicotinic receptor (Bodnar et al., 2005) and as neuroleptic compounds (Florvall \& Oegren, 1982). 2-Ethoxybenzamide, under the name ethenzamide, is a commonly used analgesicum (Darias et al., 1992).

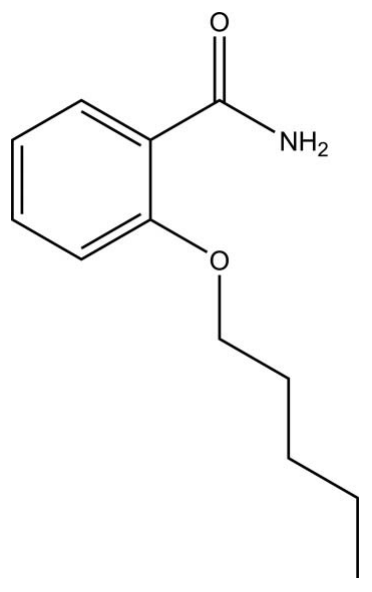

In our efforts to use 2-alkoxybenzamides as components in co-crystal formation (Aitipamula et al., 2012), we prepared the title compound, 2-pentyloxybenzamide, and report herein on its crystal structure. 2-Pentyloxybenzamide was first studied for its antipyretic and analgesic properties (Bavin et al., 1952; 


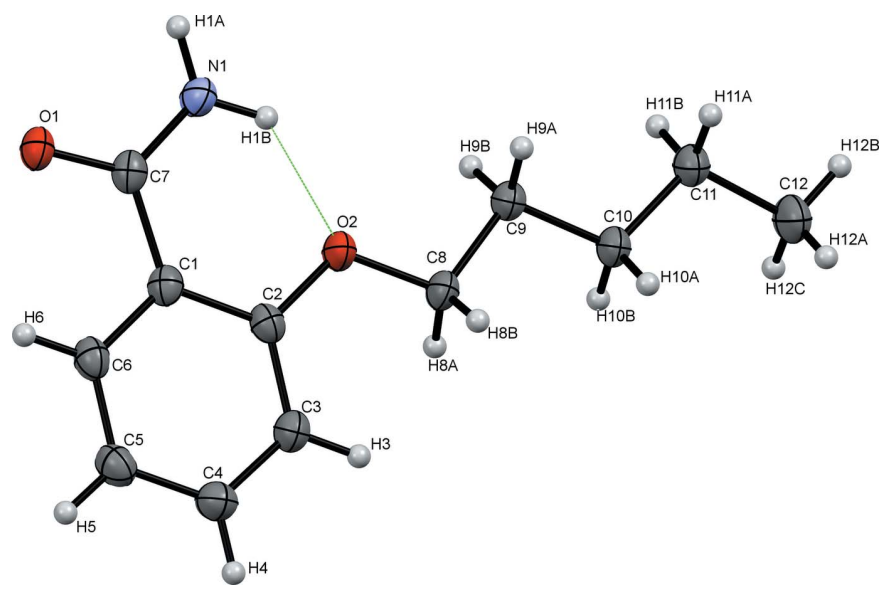

Figure 1

A view of the molecular structure of the title molecule, with atom labelling. Displacement ellipsoids are shown at the $50 \%$ probability level. The intramolecular $\mathrm{N}-\mathrm{H} \cdots \mathrm{O}$ hydrogen bond is shown as a green dashed line (see Table 1 for details).

Macrae \& Seymour, 1956). Afterwards, it was found to have antifungal activity and to be useful in the treatment of dermatomycosis (Simmonite \& Tattersall, 1962; Coates et al., 1957). Under the name pentalamide, it is still used as an ingredient in antifungal agents for topical use.

\section{Structural commentary}

In the title molecule, Fig. 1, the benzene ring is nearly coplanar with the amide group $\left[\mathrm{C} 6-\mathrm{C} 1-\mathrm{C} 7-\mathrm{O} 1=-2.48(18)^{\circ}\right]$ and the pentyloxy group $\left[\mathrm{C} 3-\mathrm{C} 2-\mathrm{O} 2-\mathrm{C} 8=0.37(18)^{\circ}\right]$. The amide $\mathrm{NH}_{2}$ group is oriented towards the ether group allowing

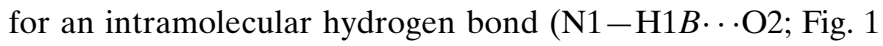
and Table 1). The latter is also present in analogous compounds, such as 3-hydroxy-2-methoxybenzamide (Wilbrand et al., 2012), 2-propoxybenzamide (Al Jasem et al.,

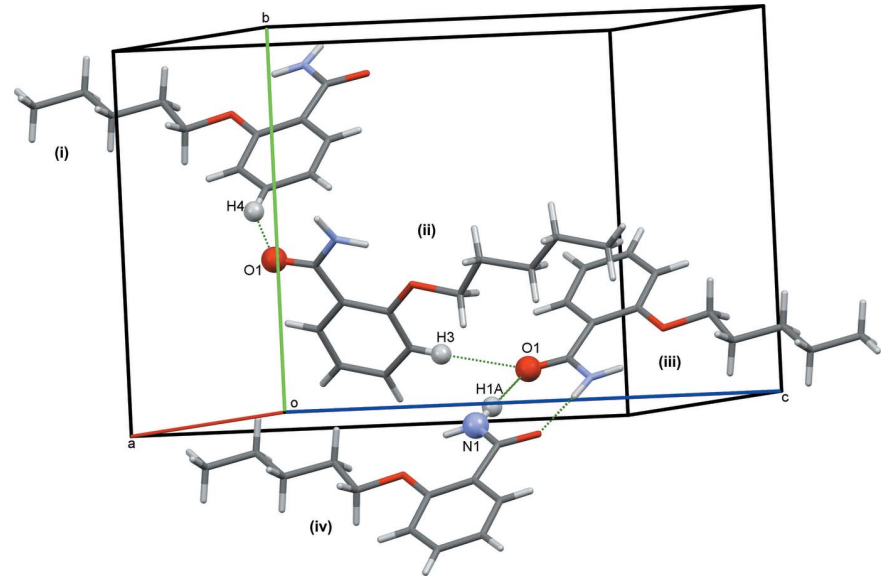

Figure 2

A partial view of the crystal packing of the title compound. The hydrogen bonds are shown as green dashed lines [see Table 1 for details; symmetry codes: (i) $-x+1, y+\frac{1}{2},-z+\frac{1}{2}$; (ii) $x, y, z$; (iii) $x,-y+\frac{1}{2}, z+\frac{1}{2}$; (iv) $-x, y-\frac{1}{2}$, $\left.-z+\frac{1}{2}\right]$
Table 1

Hydrogen-bond geometry $\left(\AA{ }^{\circ}\right)$.

$C g 1$ is the centroid of the $\mathrm{C} 1-\mathrm{C} 6$ benzene ring.

\begin{tabular}{lllll}
\hline$D-\mathrm{H} \cdots A$ & $D-\mathrm{H}$ & $\mathrm{H} \cdots A$ & $D \cdots A$ & $D-\mathrm{H} \cdots A$ \\
\hline $\mathrm{N} 1-\mathrm{H} 1 B \cdots \mathrm{O} 2$ & $0.915(17)$ & $1.921(18)$ & $2.6510(15)$ & $135.4(15)$ \\
$\mathrm{N} 1-\mathrm{H} 1 A \cdots \mathrm{O} 1^{\mathrm{i}}$ & $0.919(19)$ & $1.964(19)$ & $2.8824(15)$ & $177.8(17)$ \\
$\mathrm{C} 3-\mathrm{H} 3 \cdots \mathrm{O} 1^{\mathrm{ii}}$ & 0.93 & 2.62 & $3.546(2)$ & 178 \\
$\mathrm{C} 4-\mathrm{H} 4 \cdots 1^{\text {iii }}$ & 0.93 & 2.53 & $3.306(2)$ & 141 \\
$\mathrm{C} 11-\mathrm{H} 11 A \cdots C g 1^{\text {iv }}$ & 0.97 & 2.90 & $3.7283(16)$ & 141 \\
\hline
\end{tabular}

Symmetry codes: (i) $-x,-y+1,-z$; (ii) $x,-y+\frac{1}{2}, z+\frac{1}{2}$; (iii) $-x+1, y-\frac{1}{2},-z+\frac{1}{2}$; (iv) $x-1, y, z$.

2012) and 2-(prop-2-enyloxy)benzamide (Bugenhagen et al., 2012). 2-Ethoxybenzamide is the only studied 2-alkoxybenzamide that does not exhibit an intramolecular hydrogen bond in the single component crystal (Pagola \& Stephens, 2009). However, it shows a similar conformation to the other 2-alkoxybenzamides in the co-crystal form with thiourea (Moribe et al., 2004), and with salicylic acid (Back et al., 2012).

\section{Supramolecular features}

In the crystal, molecules are linked by pairs of $\mathrm{N}-\mathrm{H} \cdots \mathrm{O}(\mathrm{N} 1-$ $\mathrm{H} 1 A \cdots \mathrm{O} 1$ ) hydrogen bonds forming inversion dimers (Fig. 2 and Table 1). These dimers form a nested network of molecules, made of two layers, (121) and (121), which form an angle of $85.31(2)^{\circ}$ between their planes (Fig. 3). The dimers in the layers are linked by $\mathrm{C}-\mathrm{H} \cdots \mathrm{O}(\mathrm{C} 4-\mathrm{H} 4 \cdots \mathrm{O} 1)$ hydrogen bonds and $\mathrm{C}-\mathrm{H} \cdots \pi$ interactions, forming a three-dimensional framework (Fig. 3 and Table 1). Within two parallel layers, pairs of molecules lie with an offset to each other without any noticeable, direct interaction between them; the

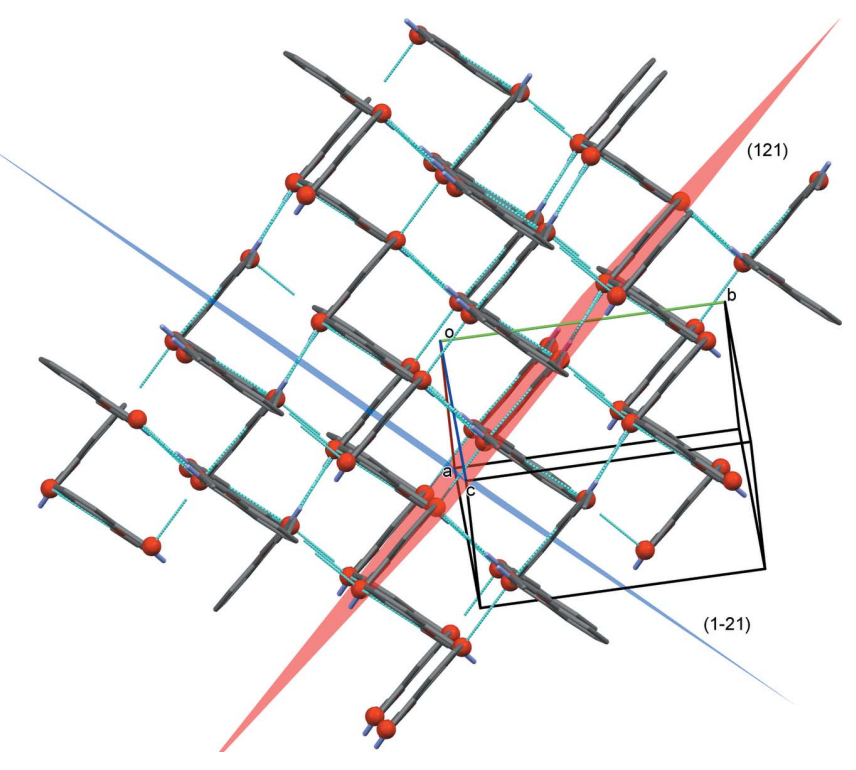

Figure 3

A view of the crystal network formed by the layers of inversion dimers in the planes (121) in red, and (1 $\overline{2} 1)$ in blue. The hydrogen bonds are shown as green dashed lines (see Table 1 for details; $\mathrm{H}$ atoms have been omitted for clarity). 
parallel layers are at a distance of 3.81 (3) $\AA$ from each other. Along the $a$ axis the pairs are ordered in two symmetry-related columns. The plane of the benzene ring $(\mathrm{C} 1-\mathrm{C} 6)$ of the 2pentyloxybenzamide forms an angle of $25.29(2)^{\circ}$ with the column axis.

\section{Database survey}

From a database survey (Cambridge Structural Database, Version 5.35, last update May 2014; Allen, 2002), the following were picked as relevant comparable structures: 3-hydroxy-2methoxybenzamide (Wilbrand et al., 2012), 2-methoxybenzamide (Moribe et al., 2006), 2-ethoxybenzamide (Pagola \& Stephens, 2009; Back et al., 2012), 2-propoxybenzamide (Al Jasem et al., 2012) and 2-(prop-2-enyloxy)benzamide (Bugenhagen et al., 2012). For 2-propoxybenzamide, a homologue of the title compound, a similar formation of inversion-related molecular pairs in the crystal was reported, hence the two compounds exhibit a similar packing. The noticeable difference between the two compounds is the larger dihedral angle between the carboxamide group and the benzene ring in 2-propoxybenzamide, $12.41(2)^{\circ}$ compared to $3.30(15)^{\circ}$ in the title compound, 2-pentyloxybenzamide. Also, the parallel layers of molecules in the title compound are further apart [separated by 3.81 (3) $\AA$ ] than is found for a similar packing of 2-propoxybenzamide [3.69 (2) $\AA$ ]. Similarly, inversion-related pairs of molecules are formed by intermolecular (amide-amide) hydrogen bonding in 2-ethoxybenzamide and 3-hydroxy-2-methoxybenzamide. As 2ethoxybenzamide exhibits no intramolecular hydrogen bonding, the freed acceptor-donor sites are used for additional intermolecular hydrogen bonding with the adjacent molecule.

In contrast, in 2-methoxybenzamide and in 2-(prop-2-enyloxy)benzamide the intermolecular $\mathrm{N}-\mathrm{H} \cdots \mathrm{O}$ hydrogen bonds involving the amide groups do not lead to pair formation but generate $C(4)$ and $R_{3}^{2}(7)$ motifs.

\section{Synthesis and crystallization}

The preparation of the title compound follows a Williamson ether synthesis using DMSO as solvent, analogous to a general procedure (Johnstone \& Rose, 1979): To powdered $\mathrm{KOH}$ $(1.12 \mathrm{~g}, 20.0 \mathrm{mmol})$ in DMSO $(18 \mathrm{ml})$ was added salicylamide $(1.37 \mathrm{~g}, 10.0 \mathrm{mmol})$, and the resulting mixture was stirred for $10 \mathrm{~min}$. at rt. Then, $n$-amyl iodide $(4.2 \mathrm{~g}, \mathrm{mmol}, 21.2 \mathrm{mmol})$ was added dropwise. The solution was stirred for $12 \mathrm{~h}$ at rt. It was then poured into water $(200 \mathrm{ml})$ and extracted with chloroform $(3 \times 75 \mathrm{ml})$. The organic phase was dried over anhydrous $\mathrm{MgSO}_{4}$, concentrated in vacuo, and the residue was subjected to column chromatography on silica gel $\left(\mathrm{CHCl}_{3} /\right.$ $\mathrm{M}^{t} \mathrm{BE} /$ hexane $v / v / v$ 1:1:1) to give the title compound (1.55 $\mathrm{g}$, $75 \%$ ) as colourless crystals (m.p. $362 \mathrm{~K})$. IR $\left(\mathrm{KBr}, \mathrm{cm}^{-1}\right)$ vmax 3434, 3168, 2948, 2868, 1664, 1593, 1387, 1232, 1164, 1018, 832, $788,765,575 ;{ }^{1} \mathrm{H}$ NMR $\left(400 \mathrm{MHz}, \mathrm{CDCl}_{3}, \delta_{\mathrm{H}}\right) 0.93\left(3 \mathrm{H}, t,{ }^{3} \mathrm{~J}=\right.$ $\left.7.2 \mathrm{~Hz}, \mathrm{CH}_{3}\right), 1.38-1.48(4 \mathrm{H}, m), 1.84-1.89(2 \mathrm{H}, m), 4.11(2 \mathrm{H}$, $\left.d,{ }^{3} J=6.4 \mathrm{~Hz}\right) ; 6.03(1 \mathrm{H}, b s, \mathrm{NH}), 6.96\left(1 \mathrm{H}, d,{ }^{3} \mathrm{~J}=8.4 \mathrm{~Hz}\right)$,
Table 2

Experimental details.

\begin{tabular}{|c|c|}
\hline \multicolumn{2}{|l|}{ Crystal data } \\
\hline Chemical formula & $\mathrm{C}_{12} \mathrm{H}_{17} \mathrm{NO}_{2}$ \\
\hline$M_{\mathrm{r}}$ & 207.27 \\
\hline Crystal system, space group & Monoclinic, $P 2_{1} / c$ \\
\hline Temperature $(\mathrm{K})$ & 100 \\
\hline$a, b, c(\AA)$ & $8.1830(2), 11.2706(2), 14.5386(4)$ \\
\hline$\beta\left(^{\circ}\right)$ & $119.696(2)$ \\
\hline$V\left(\mathrm{~A}^{3}\right)$ & $1164.76(5)$ \\
\hline$Z$ & 4 \\
\hline Radiation type & $\mathrm{Cu} K \alpha$ \\
\hline$\mu\left(\mathrm{mm}^{-1}\right)$ & 0.64 \\
\hline Crystal size $(\mathrm{mm})$ & $0.25 \times 0.19 \times 0.10$ \\
\hline \multicolumn{2}{|l|}{ Data collection } \\
\hline Diffractometer & SuperNova, Dual, $\mathrm{Cu}$ at zero, Atlas \\
\hline Absorption correction & $\begin{array}{l}\text { Multi-scan (CrysAlis PRO; } \\
\text { Agilent, 2012) }\end{array}$ \\
\hline$T_{\min }, T_{\max }$ & $0.854,1.000$ \\
\hline $\begin{array}{l}\text { No. of measured, independent and } \\
\text { observed }[I>2 \sigma(I)] \text { reflections }\end{array}$ & $6112,2268,1900$ \\
\hline$R_{\text {int }}$ & 0.022 \\
\hline$(\sin \theta / \lambda)_{\max }\left(\AA^{-1}\right)$ & 0.621 \\
\hline \multicolumn{2}{|l|}{ Refinement } \\
\hline$R\left[F^{2}>2 \sigma\left(F^{2}\right)\right], w R\left(F^{2}\right), S$ & $0.041,0.115,1.03$ \\
\hline No. of reflections & 2268 \\
\hline No. of parameters & 145 \\
\hline $\mathrm{H}$-atom treatment & $\begin{array}{l}\mathrm{H} \text { atoms treated by a mixture of } \\
\text { independent and constrained } \\
\text { refinement }\end{array}$ \\
\hline$\Delta \rho_{\max }, \Delta \rho_{\min }\left(\mathrm{e} \AA^{-3}\right)$ & $0.19,-0.22$ \\
\hline
\end{tabular}

Computer programs: CrysAlis PRO (Agilent, 2012), SHELXS97 and SHELXL97 (Sheldrick, 2008) within OLEX2 (Dolomanov et al., 2009), PLATON (Spek, 2009) and Mercury (Macrae et al., 2008).

7.03-7.07 $(1 \mathrm{H}, m), 7.42-7.46(1 \mathrm{H}, m), 7.85(1 \mathrm{H}, b s, \mathrm{NH}), 8.20$ $\left(1 \mathrm{H}, d d,{ }^{3} J=7.6 \mathrm{~Hz},{ }^{4} J=2.0 \mathrm{~Hz}\right),{ }^{13} \mathrm{C} \mathrm{NMR}(100.5 \mathrm{MHz}$, $\left.\mathrm{CDCl}_{3}, \delta_{\mathrm{C}}\right) 14.0\left(\mathrm{CH}_{3}\right), 22.4\left(\mathrm{CH}_{2}\right), 28.2\left(\mathrm{CH}_{2}\right), 28.9\left(\mathrm{CH}_{2}\right)$, $69.1\left(\mathrm{OCH}_{2}\right), 112.2(\mathrm{CH}), 120.7\left(\mathrm{C}_{\text {quat }}\right), 121.0(\mathrm{CH}), 132.5$ $(\mathrm{CH}), 133.3\left(\mathrm{C}_{\text {quat }}\right), 157.4\left(\mathrm{C}_{\text {quat }}\right), 167.2\left(\mathrm{C}_{\text {quat }}, \mathrm{CO}\right)$.

\section{Refinement}

Crystal data, data collection and structure refinement details are summarized in Table 2. All C-bound $\mathrm{H}$ atoms were placed in calculated positions and refined as riding atoms: $\mathrm{C}-\mathrm{H}$ distances of $0.95-1.00 \AA$ with $U_{\text {iso }}(\mathrm{H})=x U_{\text {eq }}(\mathrm{C})$, where $x=$ 1.5 for methyl and $=1.2$ for other $\mathrm{H}$-atoms. The $\mathrm{N}$-bound $\mathrm{H}$ atoms were located in a difference electron-density map and freely refined.

\section{References}

Agilent (2012). CrysAlis PRO. Agilent Technologies, Yarnton, England.

Aitipamula, S., Wong, A. B. H., Chow, P. S. \& \&Tan, R. B. H. (2012). CrystEngComm, 14, 8515-8524.

Al Jasem, Y., Hindawi, B. al, Thiemann, T. \& White, F. (2012). Acta Cryst. E68, o2639-o2640.

Allen, F. H. (2002). Acta Cryst. B58, 380-388.

Back, K. R., Davey, R. J., Grecu, T., Hunter, C. A. \& Taylor, L. S. (2012). Cryst. Growth Des. 12, 6110-6117.

Bavin, E. M., Macrae, F. J., Seymour, D. E. \& Waterhouse, P. D. (1952). J. Pharm. Pharmacol. 4, 872-878. 
Bodnar, A. L., Cortes-Burgos, L. A., Cook, K. K., Dinh, D. M., Groppi, V. E., Hajos, M., Higdon, N. R., Hoffmann, W. E., Hurst, R. S., Myers, J. K., Rogers, N. B., Wall, T. M., Wolfe, M. L. \& Wong, E. (2005). J. Med. Chem. 48, 905-908.

Bugenhagen, B., Al Jasem, Y., Barkhad, F., Hindawi, B. al \& Thiemann, T. (2012). Acta Cryst. E68, o3169-o3170.

Coates, L. V., Drain, D. J., Kerridge, K. A., Macrae, F. J. \& Tattersall, K. (1957). J. Pharm. Pharmacol. 9, 855-862.

Darias, V., Bravo, L., Abdallah, S. S., Sánchez Mateo, C. C., ExpósitoOrta, M. A., Lissavetsky, J. \& Manzanares, J. (1992). Arch. Pharm. 325, 83-87.

Dolomanov, O. V., Bourhis, L. J., Gildea, R. J., Howard, J. A. K. \& Puschmann, H. (2009). J. Appl. Cryst. 42, 339-341.

Florvall, L. \& Oegren, S.-O. (1982). J. Med. Chem. 1982, 25, 12801286.

Johnstone, R. A. W. \& Rose, M. E. (1979). Tetrahedron, 35, 2169 2173.

Macrae, C. F., Bruno, I. J., Chisholm, J. A., Edgington, P. R., McCabe, P., Pidcock, E., Rodriguez-Monge, L., Taylor, R., van de Streek, J. \& Wood, P. A. (2008). J. Appl. Cryst. 41, 466-470.
Macrae, F. J. \& Seymour, D. E. (1956). GB 726786 (May 1955) [Herts Pharmaceuticals Ltd.], Chem. Abstr. 50, 28262.

Monkovic, I., Willner, D., Adam, M. A., Brown, M., Crenshaw, R. R., Fuller, C. E., Juby, P. F., Luke, G. M., Matiskella, J. A. \& Montzka, T. A. (1988). J. Med. Chem. 31, 1548-1566.

Moribe, K., Tsuchiya, M., Tosuka, Y., Yamaguchi, K., Oguchi, T. \& Yamamoto, K. (2004). Chem. Pharm. Bull. 52, 524-529.

Moribe, K., Tsuchiya, M., Tozuka, Y., Yamaguchi, K., Oguchi, T. \& Yamamoto, K. (2006). J. Inclusion Phenom. Macrocycl. Chem. 54, 9-16.

Pagola, S. \& Stephens, P. W. (2009). Acta Cryst. C65, o583-o586.

Sheldrick, G. M. (2008). Acta Cryst. A64, 112-122.

Simmonite, D. \& Tattersall, K. (1962). GB 872891 (July 1961) [Smith, T. J. \& Nephew Ltd.], Chem. Abstr. 56, 18626.

Spek, A. L. (2009). Acta Cryst. D65, 148-155.

Waterbeemd, H. van de \& Testa, B. (1983). J. Med. Chem. 26, 203207.

Wilbrand, S., Neis, C. \& Hegetschweiler, K. (2012). Acta Cryst. E68, o3494. 


\section{supporting information}

Acta Cryst. (2014). E70, 231-234 [doi:10.1107/S1600536814020571]

\section{Crystal structure of 2-pentyloxybenzamide}

\section{Bernhard Bugenhagen, Yosef Al Jasem and Thies Thiemann}

\section{Computing details}

Data collection: CrysAlis PRO (Agilent, 2012); cell refinement: CrysAlis PRO (Agilent, 2012); data reduction: CrysAlis PRO (Agilent, 2012); program(s) used to solve structure: SHELXS97 (Sheldrick, 2008); program(s) used to refine structure: SHELXL97 (Sheldrick, 2008) within OLEX2 (Dolomanov et al., 2009); molecular graphics: PLATON (Spek, 2009) and Mercury (Macrae et al., 2008); software used to prepare material for publication: SHELXL97 (Sheldrick, 2008) and PLATON (Spek, 2009).

\section{2-pentyloxybenzamide}

Crystal data

$\mathrm{C}_{12} \mathrm{H}_{17} \mathrm{NO}_{2}$

$M_{r}=207.27$

Monoclinic, $P 2{ }_{1} / c$

$a=8.1830(2) \AA$

$b=11.2706(2) \AA$

$c=14.5386(4) \AA$

$\beta=119.696(2)^{\circ}$

$V=1164.76(5) \AA^{3}$

$Z=4$

\section{Data collection}

SuperNova, Dual, $\mathrm{Cu}$ at zero, Atlas diffractometer

Radiation source: SuperNova $(\mathrm{Cu}) \mathrm{X}$-ray Source

Mirror monochromator

Detector resolution: 10.4127 pixels $\mathrm{mm}^{-1}$

$\omega$ scans

Absorption correction: multi-scan

(CrysAlis PRO; Agilent, 2012)

\section{Refinement}

Refinement on $F^{2}$

Least-squares matrix: full

$R\left[F^{2}>2 \sigma\left(F^{2}\right)\right]=0.041$

$w R\left(F^{2}\right)=0.115$

$S=1.03$

2268 reflections

145 parameters

0 restraints
$F(000)=448$

$D_{\mathrm{x}}=1.182 \mathrm{Mg} \mathrm{m}^{-3}$

$\mathrm{Cu} K \alpha$ radiation, $\lambda=1.5418 \AA$

Cell parameters from 2743 reflections

$\theta=3.9-73.1^{\circ}$

$\mu=0.64 \mathrm{~mm}^{-1}$

$T=100 \mathrm{~K}$

Block, colourless

$0.25 \times 0.19 \times 0.10 \mathrm{~mm}$

$T_{\min }=0.854, T_{\max }=1.000$

6112 measured reflections

2268 independent reflections

1900 reflections with $I>2 \sigma(I)$

$R_{\text {int }}=0.022$

$\theta_{\max }=73.3^{\circ}, \theta_{\min }=5.3^{\circ}$

$h=-10 \rightarrow 5$

$k=-13 \rightarrow 14$

$l=-16 \rightarrow 18$

Primary atom site location: structure-invariant direct methods

Secondary atom site location: difference Fourier map

Hydrogen site location: inferred from neighbouring sites

$\mathrm{H}$ atoms treated by a mixture of independent and constrained refinement 
$w=1 /\left[\sigma^{2}\left(F_{\mathrm{o}}^{2}\right)+(0.0645 P)^{2}+0.2593 P\right]$

where $P=\left(F_{\mathrm{o}}^{2}+2 F_{\mathrm{c}}^{2}\right) / 3$

$(\Delta / \sigma)_{\max }<0.001$

$$
\Delta \rho_{\max }=0.19 \mathrm{e} \AA^{-3}
$$$$
\Delta \rho_{\min }=-0.22 \text { e } \AA^{-3}
$$

\section{Special details}

Geometry. All e.s.d.'s (except the e.s.d. in the dihedral angle between two 1.s. planes) are estimated using the full covariance matrix. The cell e.s.d.'s are taken into account individually in the estimation of e.s.d.'s in distances, angles and torsion angles; correlations between e.s.d.'s in cell parameters are only used when they are defined by crystal symmetry. An approximate (isotropic) treatment of cell e.s.d.'s is used for estimating e.s.d.'s involving 1.s. planes.

Refinement. Refinement of $F^{2}$ against ALL reflections. The weighted $R$-factor $w R$ and goodness of fit $S$ are based on $F^{2}$, conventional $R$-factors $R$ are based on $F$, with $F$ set to zero for negative $F^{2}$. The threshold expression of $F^{2}>\sigma\left(F^{2}\right)$ is used only for calculating $R$-factors (gt) etc. and is not relevant to the choice of reflections for refinement. $R$-factors based on $F^{2}$ are statistically about twice as large as those based on $F$, and $R$ - factors based on ALL data will be even larger.

Fractional atomic coordinates and isotropic or equivalent isotropic displacement parameters $\left(\hat{A}^{2}\right)$

\begin{tabular}{|c|c|c|c|c|}
\hline & $x$ & $y$ & $z$ & $U_{\text {iso }} * / U_{\text {eq }}$ \\
\hline $\mathrm{C} 1$ & $0.25614(18)$ & $0.29076(11)$ & $0.20599(10)$ & $0.0233(3)$ \\
\hline $\mathrm{C} 10$ & -0.14119 (19) & $0.33945(13)$ & $0.46481(11)$ & $0.0269(3)$ \\
\hline $\mathrm{C} 11$ & $-0.29380(19)$ & $0.41079(13)$ & $0.47067(11)$ & $0.0294(3)$ \\
\hline $\mathrm{C} 12$ & $-0.3051(2)$ & $0.37915(16)$ & $0.56955(12)$ & $0.0389(4)$ \\
\hline $\mathrm{C} 2$ & $0.21475(18)$ & $0.25854(12)$ & $0.28594(10)$ & $0.0240(3)$ \\
\hline $\mathrm{C} 3$ & $0.31443(19)$ & $0.16670(12)$ & $0.35597(11)$ & $0.0279(3)$ \\
\hline $\mathrm{C} 4$ & $0.45350(19)$ & $0.10654(13)$ & $0.34698(11)$ & $0.0303(3)$ \\
\hline $\mathrm{C} 5$ & $0.49609(19)$ & $0.13709(13)$ & $0.26885(11)$ & $0.0301(3)$ \\
\hline C6 & 0.39777 (18) & $0.22849(12)$ & $0.19968(10)$ & $0.0266(3)$ \\
\hline $\mathrm{C} 7$ & $0.15680(18)$ & $0.38484(11)$ & $0.12366(10)$ & $0.0232(3)$ \\
\hline $\mathrm{C} 8$ & $0.03619(19)$ & $0.28821(12)$ & $0.37434(10)$ & $0.0263(3)$ \\
\hline $\mathrm{C} 9$ & $-0.11424(18)$ & $0.36885(12)$ & $0.37063(10)$ & $0.0256(3)$ \\
\hline $\mathrm{H} 10 \mathrm{~A}$ & -0.1714 & 0.2558 & 0.4618 & $0.032 *$ \\
\hline H10B & -0.0229 & 0.3526 & 0.5295 & $0.032 *$ \\
\hline H11A & -0.4142 & 0.3950 & 0.4082 & $0.035^{*}$ \\
\hline H11B & -0.2672 & 0.4948 & 0.4717 & $0.035^{*}$ \\
\hline $\mathrm{H} 12 \mathrm{~A}$ & -0.3329 & 0.2962 & 0.5683 & $0.058^{*}$ \\
\hline H12B & -0.4027 & 0.4250 & 0.5710 & $0.058^{*}$ \\
\hline $\mathrm{H} 12 \mathrm{C}$ & -0.1869 & 0.3963 & 0.6315 & $0.058^{*}$ \\
\hline H1A & $-0.056(2)$ & $0.4934(17)$ & $0.0658(14)$ & $0.042(5)^{*}$ \\
\hline H1B & $-0.021(2)$ & $0.4242(16)$ & $0.1713(14)$ & $0.041(5)^{*}$ \\
\hline H3 & 0.2873 & 0.1458 & 0.4089 & $0.033 *$ \\
\hline $\mathrm{H} 4$ & 0.5188 & 0.0452 & 0.3937 & $0.036^{*}$ \\
\hline H5 & 0.5896 & 0.0967 & 0.2630 & $0.036^{*}$ \\
\hline H6 & 0.4270 & 0.2490 & 0.1475 & $0.032 *$ \\
\hline $\mathrm{H} 8 \mathrm{~A}$ & 0.1495 & 0.2950 & 0.4427 & $0.032 *$ \\
\hline H8B & -0.0062 & 0.2065 & 0.3650 & $0.032 *$ \\
\hline H9A & -0.2313 & 0.3570 & 0.3049 & $0.031^{*}$ \\
\hline H9B & -0.0767 & 0.4511 & 0.3742 & $0.031^{*}$ \\
\hline N1 & $0.01348(16)$ & $0.44361(11)$ & $0.12198(9)$ & $0.0282(3)$ \\
\hline $\mathrm{O} 1$ & $0.20767(12)$ & $0.40558(9)$ & $0.05777(7)$ & $0.0264(2)$ \\
\hline $\mathrm{O} 2$ & $0.07616(13)$ & $0.32058(8)$ & $0.29182(7)$ & 0.0271 (2) \\
\hline
\end{tabular}


supporting information

Atomic displacement parameters $\left(\AA^{2}\right)$

\begin{tabular}{lllllll}
\hline & $U^{11}$ & $U^{22}$ & $U^{33}$ & $U^{12}$ & $U^{13}$ & $U^{23}$ \\
\hline O1 & $0.0293(5)$ & $0.0312(5)$ & $0.0239(5)$ & $-0.0023(4)$ & $0.0171(4)$ & $0.0000(4)$ \\
O2 & $0.0312(5)$ & $0.0313(5)$ & $0.0271(5)$ & $0.0063(4)$ & $0.0207(4)$ & $0.0059(4)$ \\
N1 & $0.0343(6)$ & $0.0299(6)$ & $0.0271(6)$ & $0.0063(5)$ & $0.0204(5)$ & $0.0060(5)$ \\
C1 & $0.0233(6)$ & $0.0263(6)$ & $0.0213(6)$ & $-0.0034(5)$ & $0.0116(5)$ & $-0.0028(5)$ \\
C2 & $0.0227(6)$ & $0.0269(7)$ & $0.0250(6)$ & $-0.0002(5)$ & $0.0139(5)$ & $-0.0021(5)$ \\
C3 & $0.0293(7)$ & $0.0316(7)$ & $0.0266(7)$ & $0.0015(5)$ & $0.0168(6)$ & $0.0028(5)$ \\
C4 & $0.0302(7)$ & $0.0313(7)$ & $0.0300(7)$ & $0.0060(6)$ & $0.0153(6)$ & $0.0045(6)$ \\
C5 & $0.0279(7)$ & $0.0347(8)$ & $0.0309(7)$ & $0.0044(6)$ & $0.0169(6)$ & $-0.0018(6)$ \\
C6 & $0.0259(7)$ & $0.0331(7)$ & $0.0244(6)$ & $-0.0013(5)$ & $0.0153(6)$ & $-0.0028(5)$ \\
C7 & $0.0252(7)$ & $0.0244(6)$ & $0.0224(6)$ & $-0.0053(5)$ & $0.0135(5)$ & $-0.0046(5)$ \\
C8 & $0.0303(7)$ & $0.0300(7)$ & $0.0250(6)$ & $0.0035(5)$ & $0.0186(6)$ & $0.0055(5)$ \\
C9 & $0.0260(7)$ & $0.0296(7)$ & $0.0247(6)$ & $0.0022(5)$ & $0.0152(5)$ & $0.0034(5)$ \\
C10 & $0.0281(7)$ & $0.0318(7)$ & $0.0259(7)$ & $0.0031(6)$ & $0.0173(6)$ & $0.0039(5)$ \\
C11 & $0.0290(7)$ & $0.0357(7)$ & $0.0284(7)$ & $0.0037(6)$ & $0.0180(6)$ & $0.0032(6)$ \\
C12 & $0.0405(9)$ & $0.0513(10)$ & $0.0375(8)$ & $0.0100(7)$ & $0.0288(7)$ & $0.0064(7)$ \\
& & & & & & \\
\hline
\end{tabular}

Geometric parameters $\left(\AA,{ }^{\circ}\right)$

\begin{tabular}{llll}
\hline $\mathrm{O} 1-\mathrm{C} 7$ & $1.2412(15)$ & $\mathrm{C} 6-\mathrm{H} 6$ & 0.9300 \\
$\mathrm{O} 2-\mathrm{C} 2$ & $1.3706(15)$ & $\mathrm{C} 8-\mathrm{H} 8 \mathrm{~A}$ & 0.9700 \\
$\mathrm{O} 2-\mathrm{C} 8$ & $1.4381(14)$ & $\mathrm{C} 8-\mathrm{H} 8 \mathrm{~B}$ & 0.9700 \\
$\mathrm{~N} 1-\mathrm{C} 7$ & $1.3366(17)$ & $\mathrm{C} 8-\mathrm{C} 9$ & $1.5095(18)$ \\
$\mathrm{N} 1-\mathrm{H} 1 \mathrm{~A}$ & $0.919(19)$ & $\mathrm{C} 9-\mathrm{H} 9 \mathrm{~A}$ & 0.9700 \\
$\mathrm{~N} 1-\mathrm{H} 1 \mathrm{~B}$ & $0.915(17)$ & $\mathrm{C} 9-\mathrm{H} 9 \mathrm{~B}$ & 0.9700 \\
$\mathrm{C} 1-\mathrm{C} 2$ & $1.4099(17)$ & $\mathrm{C} 9-\mathrm{C} 10$ & $1.5272(17)$ \\
$\mathrm{C} 1-\mathrm{C} 6$ & $1.3962(18)$ & $\mathrm{C} 10-\mathrm{H} 10 \mathrm{~A}$ & 0.9700 \\
$\mathrm{C} 1-\mathrm{C} 7$ & $1.5013(18)$ & $\mathrm{C} 10-\mathrm{H} 10 \mathrm{~B}$ & 0.9700 \\
$\mathrm{C} 2-\mathrm{C} 3$ & $1.3966(19)$ & $\mathrm{C} 10-\mathrm{C} 11$ & $1.5227(18)$ \\
$\mathrm{C} 3-\mathrm{H} 3$ & 0.9300 & $\mathrm{C} 11-\mathrm{H} 11 \mathrm{~A}$ & 0.9700 \\
$\mathrm{C} 3-\mathrm{C} 4$ & $1.3853(19)$ & $\mathrm{C} 11-\mathrm{H} 11 \mathrm{~B}$ & 0.9700 \\
$\mathrm{C} 4-\mathrm{H} 4$ & 0.9300 & $\mathrm{C} 11-\mathrm{C} 12$ & $1.5278(18)$ \\
$\mathrm{C} 4-\mathrm{C} 5$ & $1.3866(19)$ & $\mathrm{C} 12-\mathrm{H} 12 \mathrm{~A}$ & 0.9600 \\
$\mathrm{C} 5-\mathrm{H} 5$ & 0.9300 & $\mathrm{C} 12-\mathrm{H} 12 \mathrm{~B}$ & 0.9600 \\
$\mathrm{C} 5-\mathrm{C} 6$ & $1.385(2)$ & $\mathrm{C} 12-\mathrm{H} 12 \mathrm{C}$ & 0.9600 \\
& & & $116.23(11)$ \\
$\mathrm{C} 1-\mathrm{C} 6-\mathrm{H} 6$ & 119.0 & $\mathrm{C} 6-\mathrm{C} 1-\mathrm{C} 7$ & $118.09(12)$ \\
$\mathrm{C} 10-\mathrm{C} 11-\mathrm{C} 12$ & $111.28(12)$ & $\mathrm{C} 6-\mathrm{C} 1-\mathrm{C} 2$ & $118.2(11)$ \\
$\mathrm{C} 10-\mathrm{C} 11-\mathrm{H} 11 \mathrm{~B}$ & 109.4 & $\mathrm{C} 7-\mathrm{N} 1-\mathrm{H} 1 \mathrm{~B}$ & $118.0(10)$ \\
$\mathrm{C} 10-\mathrm{C} 11-\mathrm{H} 11 \mathrm{~A}$ & 109.4 & $\mathrm{C} 7-\mathrm{N} 1-\mathrm{H} 1 \mathrm{~A}$ & $108.35(11)$ \\
$\mathrm{C} 10-\mathrm{C} 9-\mathrm{H} 9 \mathrm{~B}$ & 110.0 & $\mathrm{C} 8-\mathrm{C} 9-\mathrm{C} 10$ & 110.0 \\
$\mathrm{C} 10-\mathrm{C} 9-\mathrm{H} 9 \mathrm{~A}$ & 110.0 & $\mathrm{C} 8-\mathrm{C} 9-\mathrm{H} 9 \mathrm{~B}$ & 110.0 \\
$\mathrm{C} 11-\mathrm{C} 12-\mathrm{H} 12 \mathrm{C}$ & 109.5 & $\mathrm{C} 8-\mathrm{C} 9-\mathrm{H} 9 \mathrm{~A}$ & 108.5 \\
$\mathrm{C} 11-\mathrm{C} 12-\mathrm{H} 12 \mathrm{~B}$ & 109.5 & $\mathrm{C} 9-\mathrm{C} 10-\mathrm{H} 10 \mathrm{~B}$ & 108.5 \\
$\mathrm{C} 11-\mathrm{C} 12-\mathrm{H} 12 \mathrm{~A}$ & 109.5 & $\mathrm{C} 9-\mathrm{C} 10-\mathrm{H} 10 \mathrm{~A}$ & 109.7 \\
$\mathrm{C} 11-\mathrm{C} 10-\mathrm{H} 10 \mathrm{~B}$ & 108.5 & &
\end{tabular}




\begin{tabular}{|c|c|c|c|}
\hline $\mathrm{C} 11-\mathrm{C} 10-\mathrm{H} 10 \mathrm{~A}$ & 108.5 & $\mathrm{C} 9-\mathrm{C} 8-\mathrm{H} 8 \mathrm{~A}$ & 109.7 \\
\hline $\mathrm{C} 11-\mathrm{C} 10-\mathrm{C} 9$ & $114.98(11)$ & $\mathrm{H} 10 \mathrm{~A}-\mathrm{C} 10-\mathrm{H} 10 \mathrm{~B}$ & 107.5 \\
\hline $\mathrm{C} 12-\mathrm{C} 11-\mathrm{H} 11 \mathrm{~B}$ & 109.4 & $\mathrm{H} 11 \mathrm{~A}-\mathrm{C} 11-\mathrm{H} 11 \mathrm{~B}$ & 108.0 \\
\hline $\mathrm{C} 12-\mathrm{C} 11-\mathrm{H} 11 \mathrm{~A}$ & 109.4 & $\mathrm{H} 12 \mathrm{~A}-\mathrm{C} 12-\mathrm{H} 12 \mathrm{C}$ & 109.5 \\
\hline $\mathrm{C} 2-\mathrm{C} 3-\mathrm{H} 3$ & 119.9 & $\mathrm{H} 12 \mathrm{~A}-\mathrm{C} 12-\mathrm{H} 12 \mathrm{~B}$ & 109.5 \\
\hline $\mathrm{C} 2-\mathrm{C} 1-\mathrm{C} 7$ & $125.65(12)$ & $\mathrm{H} 12 \mathrm{~B}-\mathrm{C} 12-\mathrm{H} 12 \mathrm{C}$ & 109.5 \\
\hline $\mathrm{C} 2-\mathrm{O} 2-\mathrm{C} 8$ & $117.38(10)$ & $\mathrm{H} 1 \mathrm{~A}-\mathrm{N} 1-\mathrm{H} 1 \mathrm{~B}$ & $123.2(14)$ \\
\hline $\mathrm{C} 3-\mathrm{C} 4-\mathrm{C} 5$ & $120.48(13)$ & $\mathrm{H} 8 \mathrm{~A}-\mathrm{C} 8-\mathrm{H} 8 \mathrm{~B}$ & 108.2 \\
\hline $\mathrm{C} 3-\mathrm{C} 4-\mathrm{H} 4$ & 119.8 & $\mathrm{H} 9 \mathrm{~A}-\mathrm{C} 9-\mathrm{H} 9 \mathrm{~B}$ & 108.4 \\
\hline $\mathrm{C} 3-\mathrm{C} 2-\mathrm{C} 1$ & $119.99(12)$ & $\mathrm{N} 1-\mathrm{C} 7-\mathrm{C} 1$ & $119.22(11)$ \\
\hline $\mathrm{C} 4-\mathrm{C} 5-\mathrm{H} 5$ & 120.4 & $\mathrm{O} 1-\mathrm{C} 7-\mathrm{C} 1$ & $119.48(11)$ \\
\hline $\mathrm{C} 4-\mathrm{C} 3-\mathrm{H} 3$ & 119.9 & $\mathrm{O} 1-\mathrm{C} 7-\mathrm{N} 1$ & $121.29(12)$ \\
\hline $\mathrm{C} 4-\mathrm{C} 3-\mathrm{C} 2$ & $120.29(12)$ & $\mathrm{O} 2-\mathrm{C} 8-\mathrm{C} 9$ & $109.63(10)$ \\
\hline $\mathrm{C} 5-\mathrm{C} 6-\mathrm{H} 6$ & 119.0 & $\mathrm{O} 2-\mathrm{C} 8-\mathrm{H} 8 \mathrm{~B}$ & 109.7 \\
\hline $\mathrm{C} 5-\mathrm{C} 6-\mathrm{C} 1$ & $121.92(12)$ & $\mathrm{O} 2-\mathrm{C} 8-\mathrm{H} 8 \mathrm{~A}$ & 109.7 \\
\hline $\mathrm{C} 5-\mathrm{C} 4-\mathrm{H} 4$ & 119.8 & $\mathrm{O} 2-\mathrm{C} 2-\mathrm{C} 3$ & $122.35(11)$ \\
\hline $\mathrm{C} 6-\mathrm{C} 5-\mathrm{H} 5$ & 120.4 & $\mathrm{O} 2-\mathrm{C} 2-\mathrm{C} 1$ & $117.66(11)$ \\
\hline $\mathrm{C} 6-\mathrm{C} 5-\mathrm{C} 4$ & $119.22(12)$ & & \\
\hline $\mathrm{C} 1-\mathrm{C} 2-\mathrm{C} 3-\mathrm{C} 4$ & $-0.3(2)$ & $\mathrm{C} 6-\mathrm{C} 1-\mathrm{C} 2-\mathrm{O} 2$ & $179.67(11)$ \\
\hline $\mathrm{C} 2-\mathrm{C} 3-\mathrm{C} 4-\mathrm{C} 5$ & $0.3(2)$ & $\mathrm{C} 7-\mathrm{C} 1-\mathrm{C} 6-\mathrm{C} 5$ & $-177.98(12)$ \\
\hline $\mathrm{C} 2-\mathrm{C} 1-\mathrm{C} 7-\mathrm{N} 1$ & $-1.4(2)$ & $\mathrm{C} 7-\mathrm{C} 1-\mathrm{C} 2-\mathrm{C} 3$ & $178.07(12)$ \\
\hline $\mathrm{C} 2-\mathrm{C} 1-\mathrm{C} 7-\mathrm{O} 1$ & $179.44(12)$ & $\mathrm{C} 7-\mathrm{C} 1-\mathrm{C} 2-\mathrm{O} 2$ & $-2.27(19)$ \\
\hline $\mathrm{C} 2-\mathrm{C} 1-\mathrm{C} 6-\mathrm{C} 5$ & $0.3(2)$ & $\mathrm{C} 8-\mathrm{C} 9-\mathrm{C} 10-\mathrm{C} 11$ & $-178.10(12)$ \\
\hline $\mathrm{C} 2-\mathrm{O} 2-\mathrm{C} 8-\mathrm{C} 9$ & $177.89(11)$ & $\mathrm{C} 8-\mathrm{O} 2-\mathrm{C} 2-\mathrm{C} 3$ & $0.37(18)$ \\
\hline $\mathrm{C} 3-\mathrm{C} 4-\mathrm{C} 5-\mathrm{C} 6$ & $-0.1(2)$ & $\mathrm{C} 8-\mathrm{O} 2-\mathrm{C} 2-\mathrm{C} 1$ & $-179.28(11)$ \\
\hline $\mathrm{C} 4-\mathrm{C} 5-\mathrm{C} 6-\mathrm{C} 1$ & $-0.2(2)$ & $\mathrm{C} 9-\mathrm{C} 10-\mathrm{C} 11-\mathrm{C} 12$ & $-177.64(12)$ \\
\hline $\mathrm{C} 6-\mathrm{C} 1-\mathrm{C} 7-\mathrm{N} 1$ & $176.66(12)$ & $\mathrm{O} 2-\mathrm{C} 8-\mathrm{C} 9-\mathrm{C} 10$ & $-175.11(10)$ \\
\hline $\mathrm{C} 6-\mathrm{C} 1-\mathrm{C} 7-\mathrm{O} 1$ & $-2.48(18)$ & $\mathrm{O} 2-\mathrm{C} 2-\mathrm{C} 3-\mathrm{C} 4$ & $-179.95(12)$ \\
\hline $\mathrm{C} 6-\mathrm{C} 1-\mathrm{C} 2-\mathrm{C} 3$ & $0.02(19)$ & & \\
\hline
\end{tabular}

Hydrogen-bond geometry $\left(A,{ }^{\circ}\right)$

$\mathrm{Cg} 1$ is the centroid of the $\mathrm{C} 1-\mathrm{C} 6$ benzene ring.

\begin{tabular}{lllll}
\hline$D-\mathrm{H} \cdots A$ & $D-\mathrm{H}$ & $\mathrm{H} \cdots A$ & $D \cdots A$ & $D-\mathrm{H} \cdots A$ \\
\hline $\mathrm{N} 1-\mathrm{H} 1 B \cdots \mathrm{O} 2$ & $0.915(17)$ & $1.921(18)$ & $2.6510(15)$ & $135.4(15)$ \\
$\mathrm{N} 1-\mathrm{H} 1 A \cdots \mathrm{O} 1^{\mathrm{i}}$ & $0.919(19)$ & $1.964(19)$ & $2.8824(15)$ & $177.8(17)$ \\
$\mathrm{C} 3-\mathrm{H} 3 \cdots \mathrm{O} 1^{\mathrm{ii}}$ & 0.93 & 2.62 & $3.546(2)$ & 178 \\
$\mathrm{C} 4-\mathrm{H} 4 \cdots \mathrm{O} 1^{\mathrm{iii}}$ & 0.93 & 2.53 & $3.306(2)$ & 141 \\
$\mathrm{C} 11-\mathrm{H} 11 A \cdots C g 1^{\text {iv }}$ & 0.97 & 2.90 & $3.7283(16)$ & 141
\end{tabular}

Symmetry codes: (i) $-x,-y+1,-z$; (ii) $x,-y+1 / 2, z+1 / 2$; (iii) $-x+1, y-1 / 2,-z+1 / 2$; (iv) $x-1, y, z$. 